\title{
Experimental Realization of an Epsilon-Near-Zero Graded-Index Metalens at Terahertz Frequencies
}

\author{
Victor Pacheco-Peña, ${ }^{1,2}$ Nader Engheta, ${ }^{2}$ Sergei Kuznetsov, ${ }^{3,45}$ Alexandr Gentselev, ${ }^{5}$ and Miguel Beruete ${ }^{1,6, *}$ \\ ${ }^{1}$ Antennas Group-TERALAB, Universidad Pública de Navarra, Campus Arrosadía, 31006 Pamplona, Spain \\ ${ }^{2}$ Department of Electrical and Systems Engineering, University of Pennsylvania, \\ Philadelphia, Pennsylvania 19104, USA \\ ${ }^{3}$ Rzhanov Institute of Semiconductor Physics SB RAS, Novosibirsk Branch "TDIAM," \\ Lavrentiev Avenue 2/1, Novosibirsk 630090, Russian Federation \\ ${ }^{4}$ Novosibirsk State University, Pirogova Street 2, 630090 Novosibirsk, Russian Federation \\ ${ }^{5}$ Budker Institute of Nuclear Physics SB RAS, Lavrentiev Avenue 11, 630090 Novosibirsk, Russian Federation \\ ${ }^{6}$ Institute of Smart Cities, Public University of Navarra, 31006 Pamplona, Spain \\ (Received 10 April 2017; revised manuscript received 8 August 2017; published 29 September 2017)
}

\begin{abstract}
The terahertz band has been historically hindered by the lack of efficient generators and detectors, but a series of recent breakthroughs have helped to effectively close the "terahertz gap." A rapid development of terahertz technology has been possible thanks to the translation of revolutionary concepts from other regions of the electromagnetic spectrum. Among them, metamaterials stand out for their unprecedented ability to control wave propagation and manipulate electromagnetic response of matter. They have become a workhorse in the development of terahertz devices such as lenses, polarizers, etc., with fascinating features. In particular, epsilon-near-zero (ENZ) metamaterials have attracted much attention in the past several years due to their unusual properties such as squeezing, tunneling, and supercoupling where a wave traveling inside an electrically small channel filled with an ENZ medium can be tunneled through it, reducing reflections and coupling most of its energy. Here, we design and experimentally demonstrate an ENZ graded-index (GRIN) metamaterial lens operating at terahertz with a power enhancement of $16.2 \mathrm{~dB}$, using an array of narrow hollow rectangular waveguides working near their cutoff frequencies. This is a demonstration of an ENZ GRIN device at terahertz and can open the path towards other realizations of similar devices enabling full quasioptical processing of terahertz signals.
\end{abstract}

DOI: 10.1103/PhysRevApplied.8.034036

\section{INTRODUCTION}

Metamaterials have demonstrated that they allow successful control of the electromagnetic response of media, opening the gate to manipulation of wave propagation [1-14]. Within this realm, metamaterials with a relative permittivity near zero [known as epsilon near zero (ENZ)] exhibit fascinating features that have been recently studied in different frequency bands of the electromagnetic spectrum, mainly, microwaves, millimeter waves, and nearinfrared and optical frequencies [15-29]. Much of the attention is due to the fact that the waves traveling inside these media show almost "infinite" phase velocity and wavelength, with nearly uniform spatial phase variation, giving rise to unconventional phenomena such as tunneling, squeezing, and supercoupling [16]. It has been demonstrated that a simple and efficient way to emulate the response of an ENZ metamaterial is to utilize narrow hollow rectangular metallic waveguides working near their cutoff frequencies [26]. This configuration has been proposed in the design of sensors [30], control of tunneling

*Corresponding author. miguel.beruete@unavarra.es with nonlinearities [31], beam shaping [32-36], enhanced molecular fluorescence [37], and impedance-matched devices [38]. Lenses have also benefited greatly from ENZ metamaterials $[33,36]$, as they offer the possibility to tailor arbitrarily the wave front at the output by simply shaping the interface profile. As demonstrated in Ref. [33], a focus can be generated at any desired position by simply profiling the output face of a uniform ENZ lens with a concave shape. The main drawback of such structures is their bulkiness due to their profiled faces. To overcome this limitation without deteriorating their performance, ENZ graded-index (ENZ GRIN) devices have been theoretically proposed in previous works [34,35].

Even though ENZ metamaterials have been demonstrated over several spectral regimes [20,21,23,26,39], the application of these concepts to the terahertz frequency band $(0.1-10 \mathrm{THz})$ has been a major challenge due to the lack of appropriate fabrication and testing technologies in this band. Typical fabrication constraints at terahertz imposed on the structure thickness relevant for ENZ GRIN implementation are of the order of hundreds of microns, whereas the lateral dimensions of the structure are to be maintained at micrometer accuracy. Such a thickness is too small for conventional microwave techniques and too 
large for standard optical lithography. In fact, this is another important reason that has hampered the development of terahertz technologies contributing to the famous "terahertz gap" [40-45]. Even though there is still a need for several technological improvements to be fulfilled, great progress has been achieved in this frequency range to the extent that modulators [43], multiplexers [46], and tunable devices $[47,48]$ have been recently demonstrated. At the same time as the gap getting "closed," growing interest in the design of metamaterials at terahertz frequencies arises [41], and different metadevices have been recently proposed [42, 49-51] demonstrating that the design of metamaterialbased devices within this frequency range is no longer elusive.

Inspired by the exciting features of ENZ metamaterials and the importance of the terahertz frequency band, here we present an experimental demonstration of an ENZ GRIN lens operating at terahertz frequencies. The structure is designed based on the recently proposed lens reported in Ref. [34] using an array of narrow hollow rectangular waveguides working near their cutoff frequencies, thus, emulating an ENZ metamaterial (Fig. 1). It is designed to work at $0.706 \mathrm{THz}(\lambda=0.428 \mathrm{~mm})$ transforming an incident plane wave into a narrow focus situated at $6 \mathrm{~mm}(14 \lambda)$ from the output of the lens.

\section{RESULTS}

\section{A. Design of the ENZ GRIN lens}

The basic principle to emulate an effective ENZ medium using a rectangular waveguide [see Fig. 1(b)] relies on its intrinsic structural dispersion. By restricting the problem to monomode $\mathrm{TE}_{01}$ operation (only the fundamental $\mathrm{TE}_{01}$ mode of propagation), the dispersion relation of the waveguide can be written as $[26,52]$

$\beta=k_{0} \sqrt{\mu_{\text {reff }} \varepsilon_{\text {reff }}}=k_{0} \sqrt{1-\left(\frac{\pi}{k_{0} h_{y}}\right)^{2}}=k_{0} \sqrt{1-\left(\frac{f_{c}}{f}\right)^{2}}$.

Here, it is assumed that the waveguide behaves as an equivalent medium with an effective permittivity and permeability ( $\left.\mu_{\text {reff }}, \varepsilon_{\text {reff }}\right), k_{0}=2 \pi / \lambda_{0}$ is the wave number at the free-space wavelength $\lambda_{0}, h_{y}$ is the larger hollow dimension of the waveguide, $f_{c}$ is the cutoff frequency of the fundamental $\mathrm{TE}_{01}$ mode, and $f$ is the operating frequency. For this mode, $\mu_{\text {reff }}=1$ [26]. Thus, the waveguide is equivalent to an effective medium with a dispersive relative effective permittivity that can be evaluated from Eq. (1). As can be seen from this equation, near the cutoff frequency of this mode, $\varepsilon_{\text {reff }}$ is close to zero, which means that the waveguide emulates an effective ENZ medium. This performance can be observed in Fig. 1(b) where the spectral response of the waveguide is evaluated in terms of $\varepsilon_{\text {reff }}$ and $\operatorname{Re}\left(\beta / k_{0}\right)$. As it is shown, near the cutoff, the ENZ
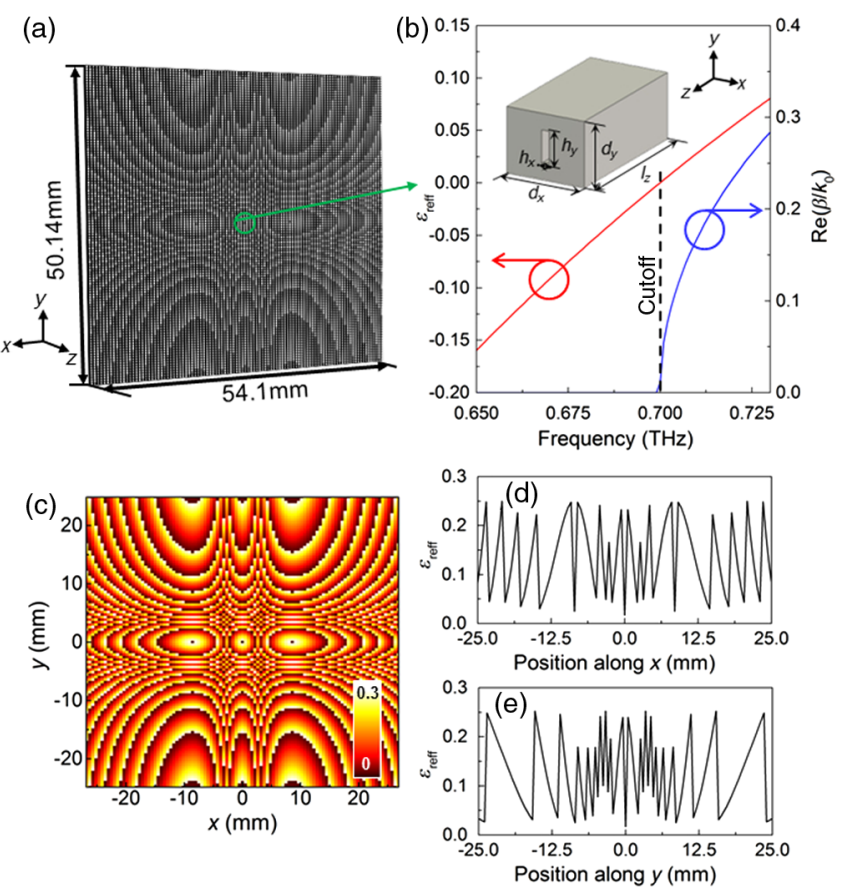

FIG. 1 (a) Schematic representation of the designed lens (artist rendition). (b) Artist presentation of the unit cell used as a central waveguide of the lens array along with the dispersive performance and $\varepsilon_{\text {reff }}$ extracted from Eq. (1). The waveguide has lateral periodicity of $d_{x}=537.5 \mu \mathrm{m}$ and $d_{y}=428.6 \mu \mathrm{m}$, hollow dimensions of $h_{x}=53.57 \mu \mathrm{m}$ and $h_{y}=214.28 \mu \mathrm{m}$, and a thickness of $l_{z}=1000 \mu \mathrm{m}$. Effective permittivity of the designed ENZ GRIN metamaterial lens: (c) complete lens, (d) along the $x$ axis at $y=0$, and (e) along the $y$ axis at $x=0$.

condition is achieved with $\operatorname{Re}\left(\beta / k_{0}\right) \approx 0$. This principle is applied here to the design of our ENZ GRIN metamaterial lens by constructing a matrix of waveguides whose dimensions are gradually varied, as we detail below.

With the GRIN technique, each waveguide of the array must introduce a prescribed phase delay $(\Delta \Phi)$ to generate a focus at the desired distance. This phase delay can be calculated by ray tracing, assuming that the lens is discretized along both $x$ and $y$ axes and can, thus, be expressed as

$$
\begin{aligned}
\Delta \Phi^{(m, n)}= & \beta^{(0,0)} l_{z}-k_{0}\left[\sqrt{L_{F}^{2}+\left(m d_{x}\right)^{2}+\left(n d_{y}\right)^{2}}-L_{F}\right] \\
& +2 \pi \alpha,
\end{aligned}
$$

where $m$ and $n$ are integers and define the discretization (i.e., each waveguide) of the array along the $x$ and $y$ axes, $\beta^{(0,0)}$ is the propagation constant of the waveguide at the center of the array [reference waveguide; see dimensions in Fig. 1(b)], $l_{z}$ is the thickness of the lens, $L_{F}$ is the focal length, $d_{x}$ and $d_{y}$ are the periods along $x$ and $y$, and $\alpha$ is an integer number $(\alpha=1,2,3 \ldots)$. As deduced from the above equation, the phase delay introduced by each waveguide 
must balance the phase difference between the output of the lens and the focus.

The larger dimension of the reference waveguide is designed to fix the cutoff at $0.7 \mathrm{THz}\left(h_{y}=\lambda_{0} / 2\right)$. Nevertheless, it has been demonstrated in previous works $[30,33,34,53]$ that the ENZ condition happens close to but not exactly at the cutoff frequency due to the finite size of the waveguide along the $z$ axis. Using a numerical simulation of the central waveguide and applying the local periodicity approximation [34,35], we find that ENZ operation occurs at $0.706 \mathrm{THz}$. Hereafter, we take this as the design frequency of the lens, although we anticipate a slight deviation from this value due to the error introduced by the local periodicity approximation (obviously, the GRIN condition implies that each waveguide of the array must have different hollow dimensions).

Under the ENZ approximation, the dimension $h_{y}{ }^{(m, n)}$ controls the required phase delay, as determined from Eqs. (1) and (2). The short side $h_{x}{ }^{(m, n)}$ provides impedance matching with free space if it is made sufficiently narrow $[34,35]$. The equation that governs this dimension assuming $\mu_{\text {reff }}=1$ is $[16,18]$

$$
h_{x}^{(m, n)}=\frac{\beta^{(m, n)} h_{y}^{(m, n)} d_{x}}{k_{0} d_{y}} .
$$

As mentioned before, Eqs. (1) and (3) are valid when working close to the cutoff frequency of the fundamental $\mathrm{TE}_{01}$ (monomode). If the design frequency is selected to be far from the cutoff, Eqs. (1) and (3) will not be valid any longer. Hence, in order to avoid undesirable responses because of a multimodal performance and to be able to engineer both the $h_{y}{ }^{(m ., n)}$ and $h_{x}{ }^{(m ., n)}$ dimensions, the ENZ region is needed. With these conditions, the ENZ GRIN metamaterial lens can be designed; see schematic in Fig. 1(a). It consists of 11817 narrow hollow rectangular waveguides $(101 \times 117$ waveguides along the $x$ and $y$ axes, respectively) with a total physical size of $54.1 \times 50.14 \times$ $1 \mathrm{~mm}^{3}(x \times y \times z)$. The hollow dimensions of each waveguide are obtained from Eqs. (1)-(3), with more details provided in Appendix A1 (Fig. 5). In Fig. 1(c), the values of the relative effective permittivity $\left(\varepsilon_{\text {reff }}\right)$ of each waveguide retrieved from Eq. (1) are presented. Additionally, we extract the values for the two principal axes $x$ and $y$ and plot them in Figs. 1(d) and 1(e). From these figures, one can observe that all waveguides work properly within the ENZ approximation, with $\varepsilon_{\text {reff }}<0.25$ in all of them. The minimum permittivity value appears for the central waveguide $\left(\varepsilon_{\text {reff }} \sim 0.017\right)$, as imposed by design. It is important to note that the ENZ GRIN metalens designed here may bring to mind classical metallic lenses [54-56]. The main difference is that classical metallic lenses suffer from Fresnel reflections which are intrinsically present because of the impedance mismatch between the metallic waveguides and free space. Similarly, common terahertz lenses are made of conventional dielectrics such as Teflon or silicon. However, their main drawbacks are their increased thickness and high impedance mismatch, respectively [56,57]. To minimize losses produced by the impedance mismatch, it is possible to apply different techniques, such as using antireflection coatings or engineering proper transmit arrays or metasurface-based lenses [58,59]. As reported in Ref. [34] and shown in Eq. (3), the ENZ GRIN metalens that we design here minimizes this problem by modulating one hollow dimension for each waveguide of the array. The present work then corresponds to the experimental proof of concept of the proposed design and more details about numerical simulations can be found in Ref. [34].

\section{B. Experimental and numerical demonstration}

To demonstrate experimentally the performance of the ENZ GRIN lens, a patented fabrication method based on a deep X-ray lithography (DXRL) technique $[60,61]$ is employed in this work for manufacturing the lens prototype. The necessity of using DXRL is explained by a relatively high aspect ratio and actual thickness of the designed structure whose production is unattainable with optical lithography [62]. In the proposed fabrication method, high-energy $x$ rays with a typical wavelength of $1 \AA$ generated by a synchrotron radiation source are used for the lithographic patterning of a poly(methyl methacrylate) (PMMA) layer followed by its entire surface metallization (see Figs. 6 and 7 for more details about the fabrication techniques). The PMMA layer has the thickness of the designed ENZ GRIN lens $(1 \mathrm{~mm})$ and plays the role of carrying polymeric substrate, while the metallized $(\mathrm{Ag} / \mathrm{Al})$ layer deposited on the PMMA surface and having typical thickness (approximately $1 \mu \mathrm{m}$ ) larger than the terahertz radiation skin depth allows the structure to act as fully metallic in terms of its electromagnetic response. The structures of this kind are referred to in the literature as "pseudometallic" $[60,61]$. It is worth noting that their fabrication is much simpler and less expensive in comparison with conventional lithography, electroplating, and molding (usually abbreviated as LIGA, the German acronym for lithographie, galvanoformung, abformung) technology [63], which is normally used for producing high-aspect-ratio entirely metallic structures (with no dielectric core inside). Figure 2(a) illustrates the fabricated lens mounted on a circular holder with a clear aperture diameter of $50 \mathrm{~mm}$. A microscope photograph and a picture taken from the numerical simulator showing nine waveguides at the center of the structure are shown in Figs. 2(b) and 2(c), respectively. From these figures, we can estimate that the fabrication is sufficiently accurate even taking into account the stringent aspect ratio of the waveguides: the narrow hollow dimension is of the order of just a few tens of microns, whereas the depth of the waveguides is $1 \mathrm{~mm}$. For the sake of completeness, a more general view of the 
(a)

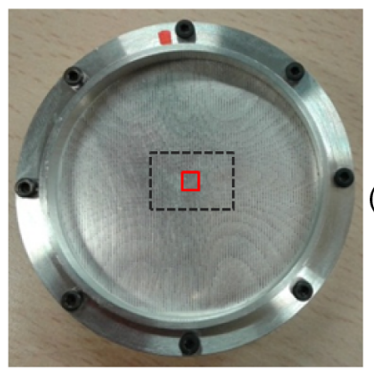

(b)

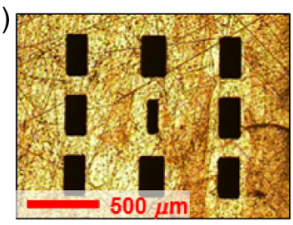

(c)
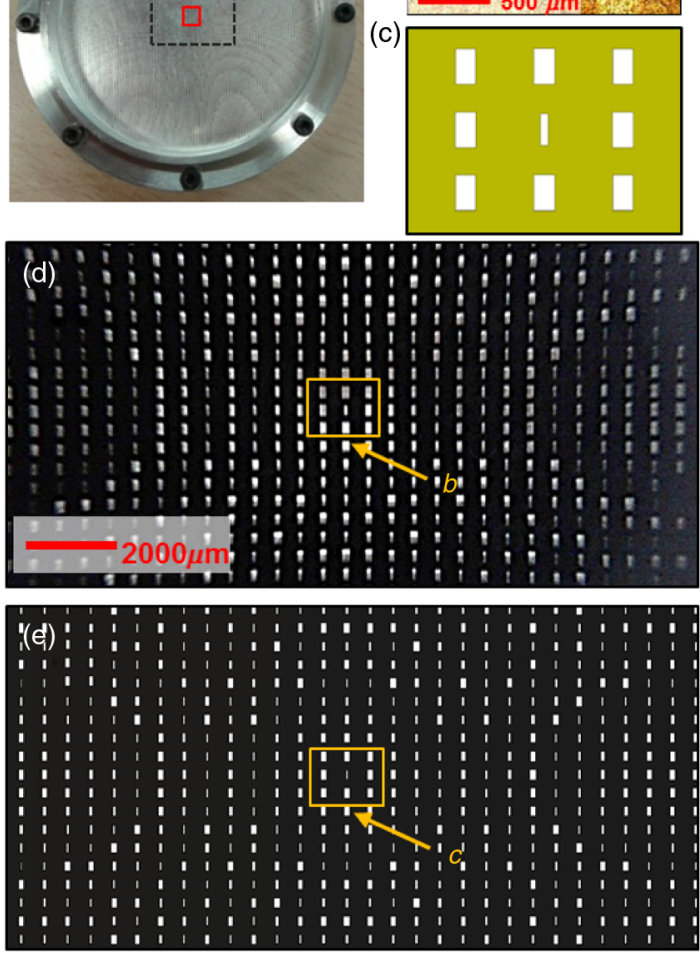

FIG. 2 (a) Full photograph of the fabricated prototype; the black dashed and red lines correspond to the enlarged pictures shown in panels (d) and (b), respectively. (b) Enlarged photograph of the nine central waveguides. (c) Schematic representation of the nine central waveguides taken from CST MicrowAVE STUdio. (d) Picture of the fabricated prototype showing several waveguides. (e) Schematic representation of the simulated ENZ GRIN metamaterial lens showing several waveguides (taken from CST MicRowAVE STUdio).

fabricated ENZ GRIN lens along with a schematic representation showing more waveguides are shown in Figs. 2(d) and 2(e) comparing the design with the fabricated prototype.

The lens is experimentally characterized with an AB Millimetre $^{\mathrm{TM}}$ vector network analyzer. First, its broadband response is evaluated by measuring the power distribution along the lens axis ( $z$ axis) from 0.630 to $0.730 \mathrm{THz}$. The lens is placed between a diagonal horn antenna (used as a source) with the electric field polarized along the $x$ direction $\left(E_{x}\right)$ and a flange-ended rectangular waveguide (used as a receiver). By shifting the receiver along the $z$ axis with micrometric precision, we measure the power distribution along the propagation direction. A full description of the setup is given in Fig. 8.

The experimental results are shown in Fig. 3(a). They are compared with numerical simulations performed with the commercial simulator CST MiCROWAVE STUDIO ${ }^{\circledR}$; see
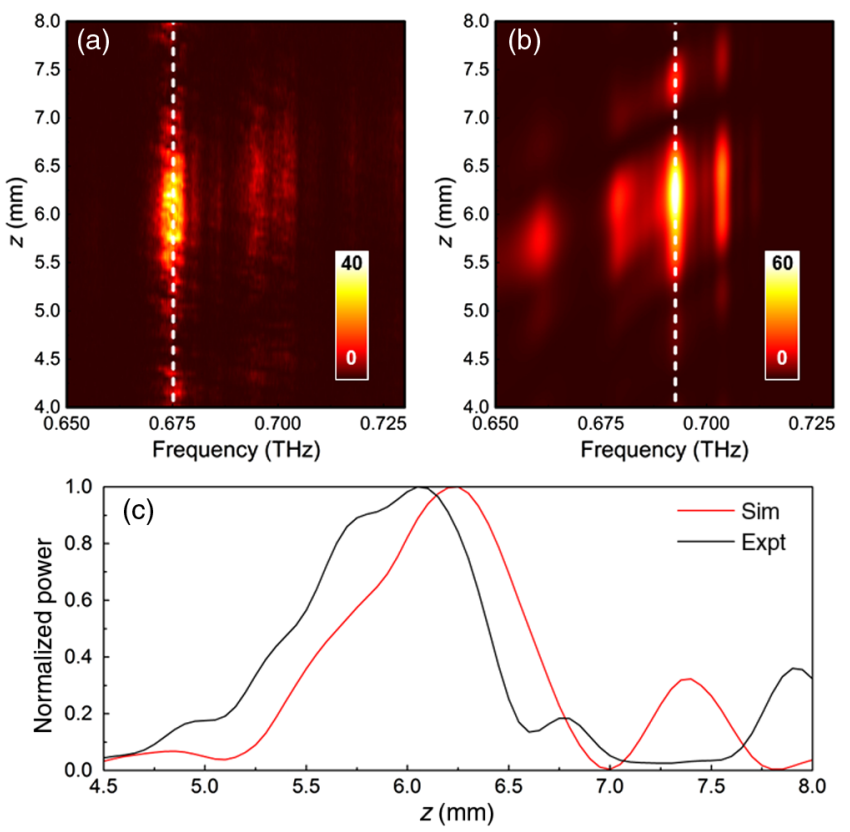

FIG. 3 Power distribution along the propagation axis of the ENZ GRIN lens vs frequency: (a) experimental and (b) numerical results. (c) Experimental (black line) and simulation (red line) results of the power distribution along the $z$ axis at the frequency of maximum focus intensity, $0.675 \mathrm{THz}$ (experiment) and $0.692 \mathrm{THz}$ (simulation). The curves in (c) are extracted from the white dashed lines in panels (a) and (b) and are normalized to the maximum of each case to better compare them.

Fig. 3(b) (for a description of the numerical setup see Appendix A4). In general, good agreement is found between the numerical and experimental results: In the experiment, the focus appears at $\left(f, L_{F}\right)=(0.675 \mathrm{THz}, 6 \mathrm{~mm})$, whereas in the simulation, it is at $\left(f, L_{F}\right)=$ $(0.692 \mathrm{THz}, 6.25 \mathrm{~mm})$. Recall that in the design, we impose $\left(f, L_{F}\right)=(0.706 \mathrm{THz}, 6 \mathrm{~mm})$. Therefore, aside from a small frequency shift, the lens has excellent performance. As mentioned earlier, the error is probably due to the local periodicity approximation, which does not totally hold in our ENZ GRIN prototype. Moreover, there are imperfections in the fabrication that might slightly alter the performance, such as waveguide cross sections being not completely rectangular and slight deviations of the hollow dimensions from the nominal values. In any case, the frequency error is very small, approximately $1.89 \%$ in the simulation and approximately $4.36 \%$ in the experiment, especially taking into account that the fabrication of this lens is highly challenging.

In Fig. 3(c), we plot the power distribution along the $z$ axis at the maximum frequency. From these curves, we can extract the depth of focus $\left(D_{F}\right.$ defined as the distance at which the power distribution has decayed half of its maximum along the propagation direction), which is another important parameter in lens performance. Again, we notice good agreement between experimental results $D_{F}=2.14 \lambda_{\exp }(0.95 \mathrm{~mm})$ and simulation results $D_{F}=$ $2.33 \lambda_{\text {sim }}(1.01 \mathrm{~mm})$. 

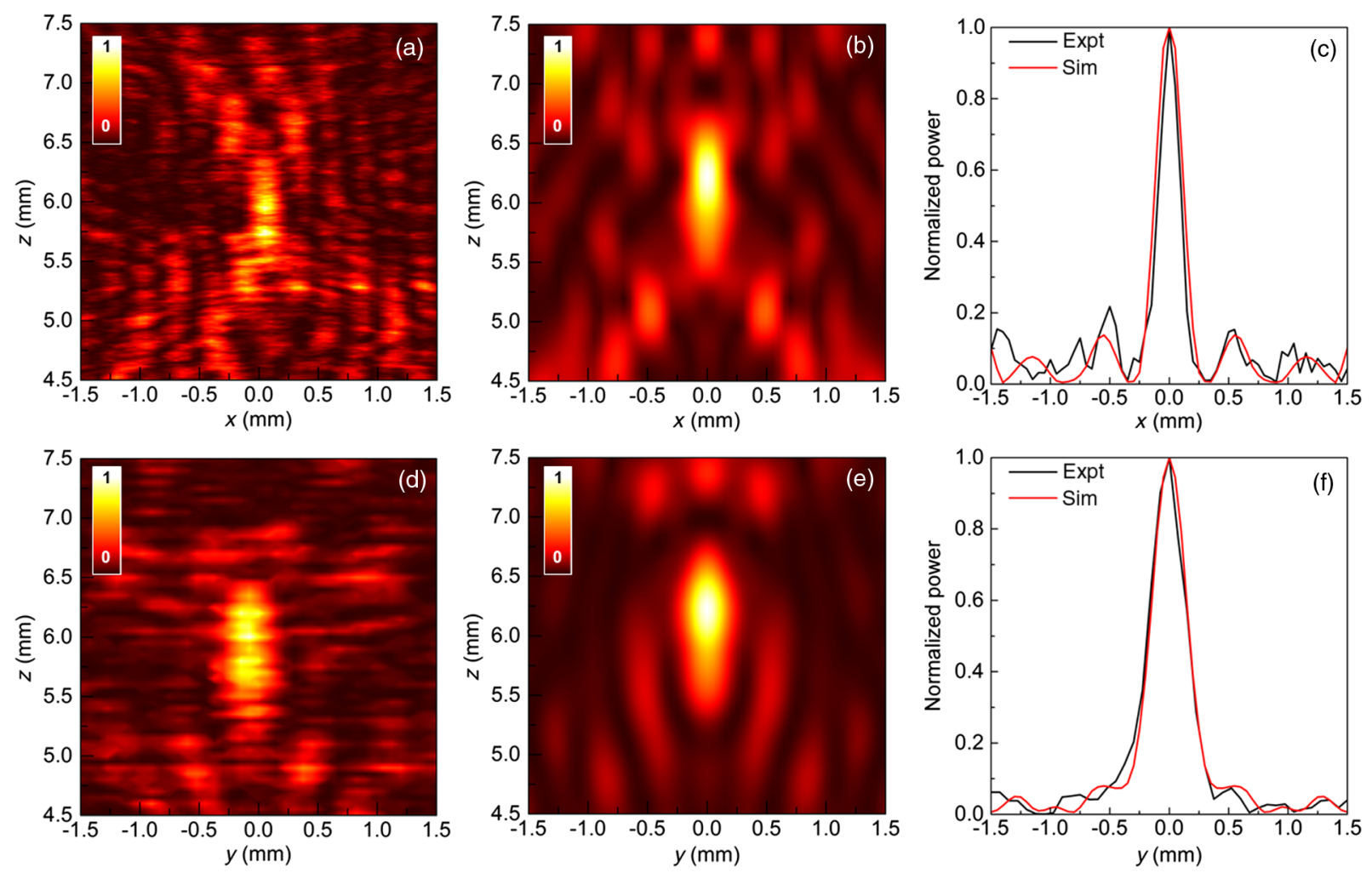

FIG. 4 Experimental and numerical results for power distribution on the focal planes: (a) and (d) are the experimental results on the $x$ - $z$ and $y-z$ planes, respectively, at $f=0.675 \mathrm{THz}$. (b) and (e) are the numerical results on the $x-z$ and $y-z$ planes, respectively, at $f=0.692 \mathrm{THz}$ where the maximum fields are obtained. This frequency is slightly different from the one in the experiment due to fabrication imperfections. In Appendix A5, we also present the numerical results for $f=0.675 \mathrm{THz}$. (c) and (f) are the experimental (black lines) and numerical (red lines) results of the power distribution at the focal position along the $x$ and $y$ axes, respectively.

For a complete characterization, the power distribution at the focal point for both principal planes ( $E$ and $H$ planes) is measured; see Appendix A4 for detailed information about the setup. The experimental results [Figs. 4(a) and 4(d)] are similar to the numerical simulations [Figs. 4(b) and 4(e)]. From these results, we observe clearly how the ENZ GRIN lens can actually focus the incident wave onto a focal spot. It produces a high power enhancement at the $L_{F}$ of 42 times $(16.23 \mathrm{~dB})$ in the experiment [69 times $(18.42 \mathrm{~dB})$ in the simulation] compared to the value without the lens. The small difference between simulation and measurement can be attributed to imperfections in fabrication such as metal roughness, waveguide dimensions, etc.

Finally, the power distribution at the focal plane along the $x$ and $y$ axes is plotted in Figs. 4(c) and 4(f). Both experimental and simulation results present similar focus profiles, from which we can obtain the full width at half maximum (FWHM) (defined as the distance along the $x$ or $y$ axes at which the power decays half of its maximum). From the experimental results, we get $\mathrm{FWHM}_{x}=0.217 \pm 0.01 \mathrm{~mm}=(0.488 \pm 0.025) \lambda_{\exp }$ and $\mathrm{FWHM}_{y}=0.337 \pm 0.01 \mathrm{~mm}=(0.76 \pm 0.025) \lambda_{\exp }$ (in the simulation, these values are $\mathrm{FWHM}_{x}=0.238 \mathrm{~mm}=$ $0.55 \lambda_{\text {sim }}$ and $\left.\mathrm{FWHM}_{y}=0.337 \mathrm{~mm}=0.78 \lambda_{\text {sim }}\right)$.

\section{CONCLUSIONS}

To sum up, we demonstrate an experimental realization of an ENZ GRIN lens at terahertz frequencies. The lens is based on a nonuniform array of narrow hollow rectangular metallic waveguides. A high power enhancement of 42 times the incident power at the focal is experimentally achieved at the frequency of $0.675 \mathrm{THz}$. The experimental values are in excellent agreement with the numerical simulations of the 3D lens. The results presented here may open doors to future work in this exciting frequency range and may lead to other terahertz devices based on ENZ media such as beam steerers, sensors, or beam hapers.

\section{ACKNOWLEDGMENTS}

The authors are grateful to the Siberian Synchrotron and Terahertz Radiation Center, Novosibirsk, Russia for providing technological infrastructure, as well as to Mr. S. G. Baev and Mr. M. A. Astafev for the help with x-ray-mask fabrication. This work is supported by the Spanish Ministerio de Economía y Competitividad under Contract No. TEC2014-51902-C2-2-R. V. P.-P. is sponsored by Spanish Ministerio de Educación, Cultura y Deporte under Grant No. FPU AP-2012-3796. S. K. acknowledges support 
from the NSU program 5-100 established by the Russian Ministry of Education and Science.

\section{APPENDIX: SUPPLEMENTARY INFORMATION}

\section{Hollow dimensions}

The hollow dimensions of the designed ENZ GRIN metalens are shown in Fig. 5. The dimensions along the $y\left(h_{y}\right)$ and $x\left(h_{y}\right)$ axes for all the waveguides of the array are calculated using Eqs. (1) and (3) and are shown in Figs. 5(a) and 5(b), respectively. Also, the hollow dimensions along the $x$ axis at $y=0$ [white dashed lines of Figs. 5(a) and 5(b)] are shown in Fig. 5(d) to better appreciate these results. As we explain in the main text, each waveguide of the array should introduce a phase delay in order to produce a focus at the output of the lens. This phase delay is controlled by changing the hollow dimension $h_{y}$. For the sake of completeness, the cutoff frequency of the fundamental mode for each waveguide is shown in Fig. 5(c) calculated using Eq. (1). To better visualize these results, the cutoff frequency along the $x$ axis at $y=0$ is shown in Fig. 5(e).

\section{Fabrication techniques}

The ENZ GRIN lens prototype is manufactured using the technological facilities of the Siberian Synchrotron and Terahertz Radiation Center, Novosibirsk, Russia. The fabrication method is based on a DXRL technique and implies the use of high-energy $\mathrm{x}$ rays produced by a synchrotron radiation source at the VEPP-3 storage ring for radiation treatment. The X-ray spectrum of the source is concentrated near the wavelength of $1 \AA$ that allows such "hard" radiation to penetrate deeply into thick layers of $\mathrm{x}$-ray-sensitive resists. Using an $\mathrm{x}$-ray-contrast mask (x mask) enables us to form patterned high-aspect microstructures having through holes with vertical walls.

The three basic stages of the fabrication process are shown in Fig. 6 . First, the polished PMMA $\left[\varepsilon_{\text {PMMA }} \approx 2.58\right.$ $\left(1-j 5 \times 10^{-2}\right)$ ] layer $1 \pm 0.01 \mathrm{~mm}$ thick, which serves both as the $\mathrm{x}$-ray-sensitive and the lens-core material, is
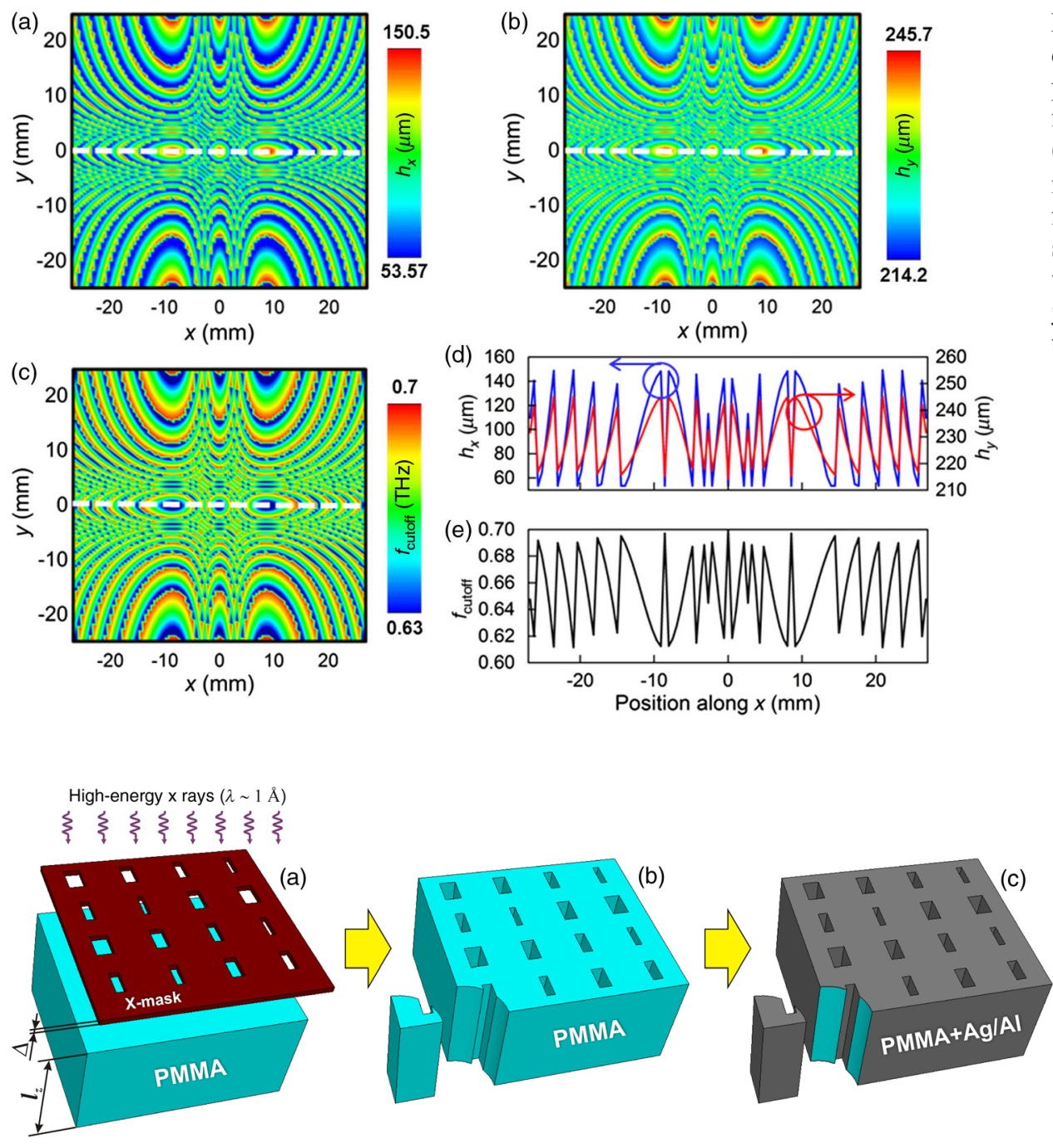

FIG. 5 Hollow dimensions of the designed ENZ GRIN lens: (a) contour plot showing the value of $h_{x}$ as a function of the waveguide position $(x, y)$. (b) Idem for $h_{y}$. (c) Cutoff frequency of the fundamental mode for all the waveguides. Hollow dimensions (d) and cutoff frequency for the waveguides located along the $x$ axis at $y=0$. These values are obtained from the white dashed line of panels (a)-(c).
FIG. 6 Basic fabrication stages for the ENZ GRIN lens: (a) irradiation of the PMMA layer by hard $\mathrm{x}$ rays through the $\mathrm{x}$ mask. (b) Chemical development of the patterned polymeric structure. (c) Metallization of the entire dielectric surface via depositing thin $\mathrm{Ag} / \mathrm{Al}$ layers. The structural slice is introduced in (b) and (c) to show that PMMA is patterned through, and all the waveguide holes have vertical walls. 

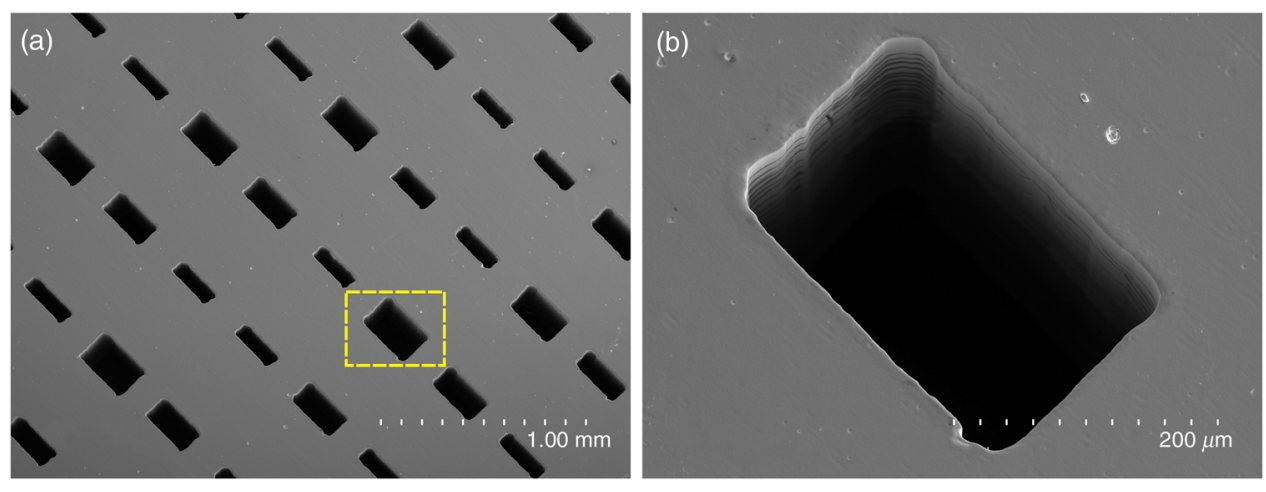

FIG. 7 SEM images of the fabricated ENZ GRIN lens structure: (a) smaller scale; (b) larger scale. The geometry of the waveguide holes slightly deviates from the ideal rectangular geometry of the designed holes that originates from some imperfection of the X-ray mask upon its fabrication by laser micromachining. exposed to $\mathrm{x}$ rays through the $\mathrm{x}$ mask [Fig. 6(a)]. The $\mathrm{x}$ mask replicates the geometry of the designed ENZ GRIN lens and is made as a patterned brass foil with thickness $\Delta=50 \pm 1 \mu \mathrm{m}$ produced in advance via laser micromachining. At the second stage, due to the radiation damage of molecular chains in PMMA upon its x-ray treatment, the irradiated parts of the PMMA layer are removed by chemical development [Fig. 6(b)]. Finally, the entire surface of the patterned polymeric structure including inner walls of the waveguide holes is metallized via chemical deposition of a silver layer $0.1 \mu \mathrm{m}$ thick, which is further "enhanced" through magnetron sputtering of additional silver and aluminum layers with the total thickness of $1 \mu \mathrm{m}$ to form the highly conductive corrosion-resistant metallic coating [Fig. 6(c)].

Note that in the described fabrication technology, the quality of the $\mathrm{x}$-mask pattern is estimated to be the main factor limiting the ENZ GRIN lens performance. Herein, we employ a homemade laser micromachining system based on a 10-ns-pulse Nd:YAG laser $\left(\lambda_{0}=1064 \mathrm{~nm}\right.$, $10 \mathrm{kHz}, 10 \mathrm{~W})$ providing the diameter of $10 \mu \mathrm{m}$ for the laser beam focused onto the brass foil. The system exhibits the typical accuracy of $\pm 3-5 \mu \mathrm{m}$ for irregularity of the hole's edge upon laser cutting, though somewhat larger geometrical deviations are tracked in fabrication (Fig. 7). It is noteworthy that such deviations do not cause a noticeable degradation of the ENZ GRIN lens performance, as we describe in the main text.

\section{Experimental setup}

The experimental measurements are carried out using an $\mathrm{AB}$ Millimetre quasioptical vector network analyzer. A general sketch of the experimental setup is shown in Fig. 8. The ENZ GRIN lens is placed in front of a standard diagonal horn antenna (WR-1.2) at $250 \mathrm{~mm}$ (approximately $588 \lambda$ at the design frequency of $0.706 \mathrm{THz}$ ). On the opposite side, a flange-ended rectangular waveguide (WR-1.2) is used as a receiver. It is placed on a translation stage in order to scan the power distribution near the focal plane. To characterize the broadband response of the lens shown in Fig. 3(a), the receiver is placed just at $x=y=0$ and is moved along $z$ from 4 to $8 \mathrm{~mm}$ with a very fine step of $0.05 \mathrm{~mm}$ (approximately $0.117 \lambda$ at the design frequency). With this configuration, the power distribution at each position is measured from 0.630 to $0.730 \mathrm{THz}$ with a high-resolution step of $0.0002 \mathrm{THz}(200 \mathrm{MHz})$. For the focal planes shown in Figs. 4(a) and 4(d), the receiver is moved on the $x-z$ and $y-z$ planes in order to experimentally characterize the focusing properties of the lens on the $E$ and $H$ planes, respectively. A high-resolution measurement is performed by moving the receiver from -1.5 to $1.5 \mathrm{~mm}$ with a step of $0.01 \mathrm{~mm}(0.024 \lambda)$ along the $x$ and $y$ axes and from 4.5 to $7.5 \mathrm{~mm}$ along the $z$ axis with a step $0.05 \mathrm{~mm}(0.117 \lambda)$.

\section{Numerical evaluation of the ENZ GRIN metalens}

The numerical analysis of the ENZ GRIN lens is performed using the transient solver of the commercial software CST Microwave Studio. For all the simulations, the full ENZ GRIN lens is simulated. A horizontally polarized plane wave $\left(E_{x}\right)$ is used to illuminate the lens from its back using open + add space (i.e., perfectly matched layers) boundary conditions in all planes. Electric and magnetic symmetry

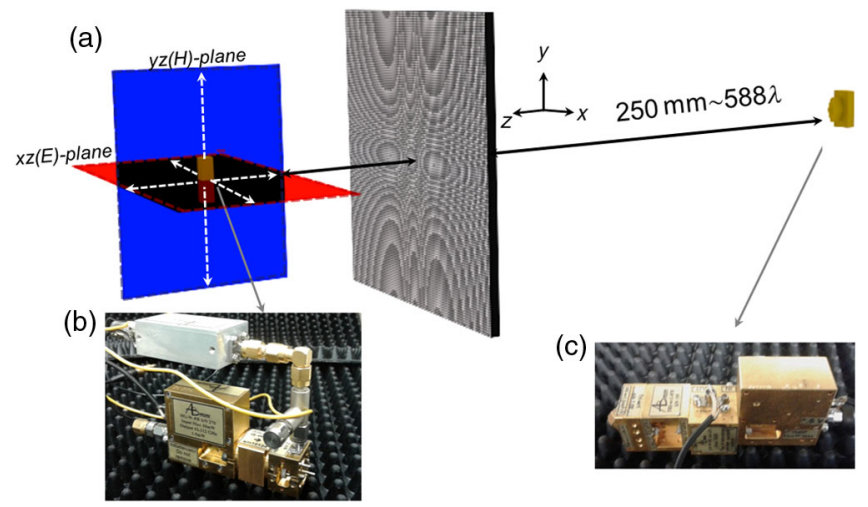

FIG. 8 Schematic representation of the setup used to characterize experimentally the performance of the ENZ GRIN lens. (a) Artist figure showing the lens placed in the middle of a diagonal horn antenna (source) and a flange-ended waveguide (receiver). The latter is placed on a translational platform in order to record the power distribution on the focal planes along the $x, y$, and $z$ axes from 0.630 to $0.730 \mathrm{THz}$. (b) Photograph of the receiver. (c) Photograph of the transmitter. 

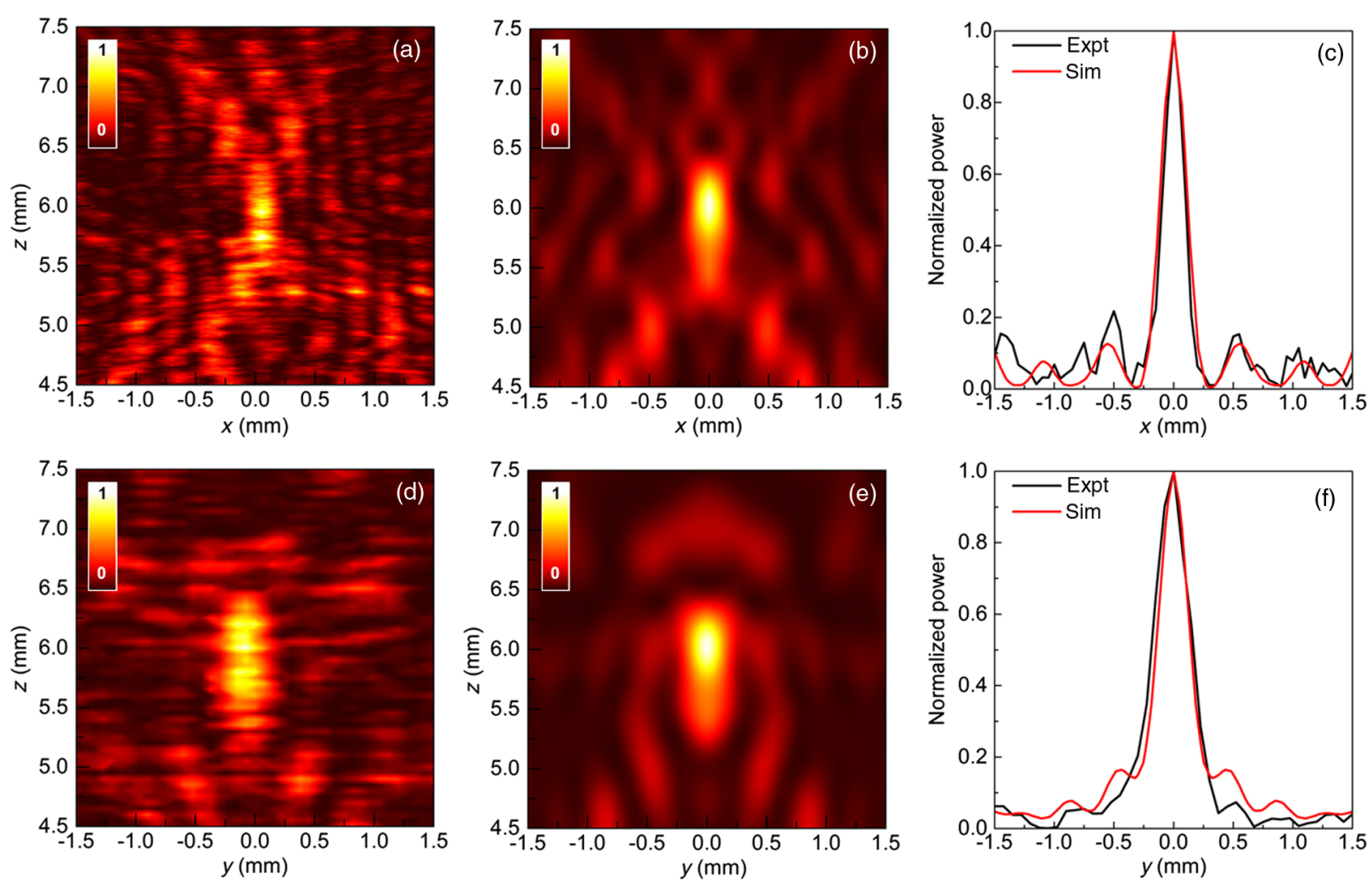

FIG. 9 Experimental and numerical results of the power distribution on the focal planes at 0.675 THz. (a),(d) experimental results on the $x-z$ and $y-z$ planes, respectively; (b),(e) numerical results on the $x-z$ and $y-z$ planes, respectively. (c),(f) experimental (black lines) and numerical (red lines) results of the power distribution at the focal position along the $x$ and $y$ axes, respectively.

planes are defined in the $y-z$ and $x-z$ planes, respectively, in order to reduce the simulation burden. Aluminum is used as the material of the lens with a conductivity of $\sigma=3.56 \times 10^{7} \mathrm{~S} / \mathrm{m}$. A very fine meshing is used with a minimum and maximum mesh size of 0.0205 and $0.101 \mathrm{~mm}$, respectively. With this configuration, the lens is simulated using a total number of approximately $82 \times 10^{6}$ cells. To characterize the broadband response of the lens, as shown in Fig. 3(b), electric and magnetic probes are placed along the $z$ axis (at $x=y=0$ ) to record the field distributions at the same positions as in the experiment. Likewise, the frequency response is obtained in each position with the same spectral range and steps used in the experiments. Finally, the broadband power distribution is calculated using the $x$ and $y$ components of the steady-state electric and magnetic field components, respectively, as $1 / 2\left(\left|E_{x}\right|\left|H_{y}\right|\right)$ After evaluating the broadband response, a second simulation of the ENZ GRIN lens is performed to calculate the 3D power distribution in both principal planes at the operation frequency corresponding to the results shown in Figs. 4(b) and 4(e).

\section{Experimental and numerical results of the power distribution at $0.675 \mathrm{THz}$}

The experimental and numerical results of the power distribution on both $x-z$ and $y-z$ focal planes at $f=$ $0.675 \mathrm{THz}$ and $f=0.692 \mathrm{THz}$, respectively, are shown and discussed in Fig. 4. These frequencies are selected by extracting the maximum field distribution from the spectral response shown in Figs. 3(a) and 3(b). To further evaluate the response of the ENZ GRIN lens, it is also interesting to evaluate its performance at the same frequency. For the sake of completeness, the numerical results of the power distribution on both $x-z$ and $y-z$ focal planes are shown in Fig. 9 at the frequency of maximum field from the experiment $(f=0.675)$. From these results, we get $\mathrm{FWHM}_{x}=0.242 \mathrm{~mm}=0.54 \lambda$ and $\mathrm{FWHM}_{y}=0.31 \mathrm{~mm}=$ $0.7 \lambda$, which are in agreement with the experimental results obtained from Figs. 4(a) and 4(d) with values of FWHM $_{x}=$ $0.217 \pm 0.1 \mathrm{~mm}=(0.488 \lambda \pm 0.025) \lambda$ and $\mathrm{FWHM}_{y}=$ $0.337 \pm 0.1 \mathrm{~mm}=(0.76 \lambda \pm 0.025) \lambda$.

[1] V. G. Veselago, The electrodynamics of substances with simultaneously negative values of $\varepsilon$ and $\mu$, Sov. Phys. Usp. 10, 509 (1968).

[2] C. G. Parazzoli, R. B. Greegor, J. A. Nielsen, M. A. Thompson, K. Li, A. M. Vetter, M. H. Tanielian, and D. C. Vier, Performance of a negative index of refraction lens, Appl. Phys. Lett. 84, 3232 (2004).

[3] R. A. Shelby, D. R. Smith, and S. Schultz, Experimental verification of a negative index of refraction, Science 292, 77 (2001). 
[4] G. Dolling, C. Enkrich, M. Wegener, C. M. Soukoulis, and S. Linden, Simultaneous negative phase and group velocity of light in a metamaterial., Science 312, 892 (2006).

[5] H. Liu and P. Lalanne, Microscopic theory of the extraordinary optical transmission, Nature (London) 452, 728 (2008).

[6] L. Solymar and E. Shamonina, Waves in Metamaterials (Oxford University Press, New York, 2009).

[7] Ernst Jan R. Vesseur, T. Coenen, H. Caglayan, N. Engheta, and A. Polman, Experimental Verification of $n=0$ Structures for Visible Light, Phys. Rev. Lett. 110, 013902 (2013).

[8] P. Moitra, Y. Yang, Z. Anderson, I. I. Kravchenko, D. P. Briggs, and J. Valentine, Realization of an all-dielectric zero-index optical metamaterial, Nat. Photonics 7, 791 (2013).

[9] C. Della Giovampaola and N. Engheta, Digital metamaterials, Nat. Mater. 13, 1115 (2014).

[10] F. Zhou, W. Cao, B. Dong, T. Reissman, W. Zhang, and C. Sun, Additive manufacturing of a 3D terahertz gradientrefractive index lens, Adv. Opt. Mater. 4, 1034 (2016).

[11] Y. Li, I. Liberal, C. Della Giovampaola, and N. Engheta, Waveguide metatronics: Lumped circuitry based on structural dispersion, Sci. Adv. 2, e1501790 (2016).

[12] A. Silva, F. Monticone, G. Castaldi, V. Galdi, A. Alù, and N. Engheta, Performing mathematical operations with metamaterials, Science 343, 160 (2014).

[13] N. I. Zheludev and Y. S. Kivshar, From metamaterials to metadevices, Nat. Mater. 11, 917 (2012).

[14] R. Maas, J. van de Groep, and A. Polman, Planar metal/ dielectric single-periodic multilayer ultraviolet flat lens, Optica 3, 592 (2016).

[15] M. Silveirinha and N. Engheta, Tunneling of Electromagnetic Energy through Subwavelength Channels and Bends using $\varepsilon$-Near-Zero Materials, Phys. Rev. Lett. 97, 157403 (2006).

[16] M. Silveirinha and N. Engheta, Theory of supercoupling, squeezing wave energy, and field confinement in narrow channels and tight bends using $\varepsilon$ near-zero metamaterials, Phys. Rev. B 76, 245109 (2007).

[17] N. Engheta, Circuits with light at nanoscales: Optical nanocircuits inspired by metamaterials, Science $\mathbf{3 1 7}$, 1698 (2007).

[18] A. Alù, M. G. Silveirinha, and N. Engheta, Transmissionline analysis of $\varepsilon$-near-zero-filled narrow channels, Phys. Rev. E 78, 016604 (2008).

[19] R. J. Pollard, A. Murphy, W. R. Hendren, P. R. Evans, R. Atkinson, G. A. Wurtz, A. V. Zayats, and V. A. Podolskiy, Optical Nonlocalities and Additional Waves in EpsilonNear-Zero Metamaterials, Phys. Rev. Lett. 102, 127405 (2009).

[20] R. Maas, J. Parsons, N. Engheta, and A. Polman, Experimental realization of an epsilon-near-zero metamaterial at visible wavelengths, Nat. Photonics 7, 907 (2013).

[21] X. Yang, C. Hu, H. Deng, D. Rosenmann, A. David, and J. Gao, Experimental demonstration of near-infrared epsilonnear-zero multilayer metamaterial slabs, Opt. Express 21, 23631 (2013).

[22] F. J. Rodríguez-Fortuño, A. Vakil, and N. Engheta, Electric Levitation Using $\in$-Near-Zero Metamaterials, Phys. Rev. Lett. 112, 033902 (2014).
[23] N. Kinsey, C. DeVault, J. Kim, M. Ferrera, V. M. Shalaev, and A. Boltasseva, Epsilon-near-zero Al-doped $\mathrm{ZnO}$ for ultrafast switching at telecom wavelengths, Optica 2, 616 (2015).

[24] C. Rizza, A. Di Falco, M. Scalora, and A. Ciattoni, OneDimensional Chirality: Strong Optical Activity in EpsilonNear-Zero Metamaterials, Phys. Rev. Lett. 115, 057401 (2015).

[25] N. Engheta, Pursuing near-zero response, Science 340, 286 (2013).

[26] B. Edwards, A. Alù, M. Young, M. Silveirinha, and N. Engheta, Experimental Verification of Epsilon-Near-Zero Metamaterial Coupling and Energy Squeezing Using a Microwave Waveguide, Phys. Rev. Lett. 100, 033903 (2008).

[27] A. Ciattoni, C. Rizza, A. E. Marini, A. Di Falco, D. Faccio, and M. Scalora, Enhanced nonlinear effects in pulse propagation through epsilon-near-zero media, Laser Photonics Rev. 10, 517 (2016).

[28] V. Pacheco-Peña, M. Navarro-Cía, and M. Beruete, Epsilonnear-zero metalenses operating in the visible, Opt. Laser Technol. 80, 162 (2016).

[29] A. Ciattoni, A. Marini, and C. Rizza, Efficient Vortex Generation in Subwavelength Epsilon-Near-Zero Slabs, Phys. Rev. Lett. 118, 104301 (2017).

[30] A. Alù and N. Engheta, Dielectric sensing in $\epsilon$-near-zero narrow waveguide channels, Phys. Rev. B 78, 045102 (2008).

[31] D. A. Powell, A. Alù, B. Edwards, A. Vakil, Y. S. Kivshar, and N. Engheta, Nonlinear control of tunneling through an epsilon-near-zero channel, Phys. Rev. B 79, 245135 (2009).

[32] A. Alù, M. G. Silveirinha, A. Salandrino, and N. Engheta, Epsilon-near-zero metamaterials and electromagnetic sources: Tailoring the radiation phase pattern, Phys. Rev. B 75, 155410 (2007).

[33] M. Navarro-Cía, M. Beruete, M. Sorolla, and N. Engheta, Lensing system and Fourier transformation using epsilonnear-zero metamaterials, Phys. Rev. B 86, 165130 (2012).

[34] V. Torres, V. Pacheco-Peña, P. Rodríguez-Ulibarri, M. Navarro-Cía, M. Beruete, M. Sorolla, and N. Engheta, Terahertz epsilon-near-zero graded-index lens, Opt. Express 21, 9156 (2013).

[35] V. Pacheco-Peña, V. Torres, M. Beruete, M. Navarro-Cía, and N. Engheta, $\varepsilon$-near-zero (ENZ) graded index quasioptical devices: Steering and splitting millimeter waves, J. Opt. 16, 094009 (2014).

[36] V. Torres, B. Orazbayev, V. Pacheco-Peña, J. Teniente, M. Beruete, M. Navarro-Cía, M. S. Ayza, and N. Engheta, Experimental demonstration of a millimeter-wave metallic ENZ lens based on the energy squeezing principle, IEEE Trans. Antennas Propag. 63, 231 (2015).

[37] A. Alù and N. Engheta, Boosting Molecular Fluorescence with a Plasmonic Nanolauncher, Phys. Rev. Lett. 103, 043902 (2009).

[38] A. Alù and N. Engheta, Coaxial-to-waveguide matching with $\varepsilon$-near-zero ultranarrow channels and bends, IEEE Trans. Antennas Propag. 58, 328 (2010).

[39] J. Gao, L. Sun, H. Deng, C. J. Mathai, S. Gangopadhyay, and X. Yang, Experimental realization of epsilon-near-zero 
metamaterial slabs with metal-dielectric multilayers, Appl. Phys. Lett. 103, 051111 (2013).

[40] P. H. Siegel, Terahertz technology, IEEE Trans. Microwave Theory Tech. 50, 910 (2002).

[41] B. Ferguson and X.-C. Zhang, Materials for terahertz science and technology, Nat. Mater. 1, 26 (2002).

[42] H. T. Chen, W. J. Padilla, J. M. O. Zide, A. C. Gossard, A. J. Taylor, and R. D. Averitt, Active terahertz metamaterial devices, Nature (London) 444, 597 (2006).

[43] H.-T. Chen, W. J. Padilla, M. J. Cich, A. K. Azad, R. D. Averitt, and A. J. Taylor, A metamaterial solid-state terahertz phase modulator, Nat. Photonics 3, 148 (2009).

[44] L. Ho, M. Pepper, and P. Taday, Terahertz spectroscopy: Signatures and fingerprints, Nat. Photonics 2, 541 (2008).

[45] P. Rodríguez-Ulibarri, S. A. Kuznetsov, and M. Beruete, Wide angle terahertz sensing with a cross-dipole frequency selective surface, Appl. Phys. Lett. 108, 111104 (2016).

[46] N. J. Karl, R. W. Mckinney, Y. Monnai, R. Mendis, and D. M. Mittleman, Frequency-division multiplexing in the terahertz range using a leaky-wave antenna, Nat. Photonics 9, 717 (2015).

[47] B. Scherger, C. Jördens, and M. Koch, Variable-focus terahertz lens, Opt. Express 19, 4528 (2011).

[48] X. Wang, Z. Xie, W. Sun, S. Feng, Y. Cui, J. Ye, and Y. Zhang, Focusing and imaging of a virtual all-optical tunable terahertz Fresnel zone plate, Opt. Lett. 38, 4731 (2013).

[49] H.-T. Chen, J. F. O'Hara, A. K. Azad, A. J. Taylor, R. D. Averitt, D. B. Shrekenhamer, and W. J. Padilla, Experimental demonstration of frequency-agile terahertz metamaterials, Nat. Photonics 2, 295 (2008).

[50] S. H. Lee, H. D. Kim, H. J. Choi, B. Kang, Y. R. Cho, and B. Min, Broadband modulation of terahertz waves with nonresonant graphene meta-devices, IEEE Trans. Terahertz Sci. Technol. 3, 764 (2013).

[51] C. Rizza, A. Ciattoni, L. Columbo, M. Brambilla, and F. Prati, Terahertz optically tunable dielectric metamaterials without microfabrication, Opt. Lett. 38, 1307 (2013).
[52] W. Rotman, Plasma simulation by artificial dielectrics and parallel-plate media, IRE Trans. Antennas Propag. 10, 82 (1962).

[53] J. Neu, R. Beigang, and M. Rahm, Metamaterial-based gradient index beam steerers for terahertz radiation, Appl. Phys. Lett. 103, 041109 (2013).

[54] W. E. Kock, Metal-lens antennas, Proc. IRE 34, 828 (1946).

[55] H. D. Hristov, Zone Plate Lenses and Antennas (Artech House, Norwood, MA, 2000).

[56] R. Mendis, M. Nagai, Y. Wang, N. Karl, and D. M. Mittleman, Terahertz artificial dielectric lens, Sci. Rep. 6, 23023 (2016).

[57] N. Llombart, G. Chattopadhyay, A. Skalare, and I. Mehdi, Novel terahertz antenna based on a silicon lens fed by a leaky wave enhanced waveguide, IEEE Trans. Antennas Propag. 59, 2160 (2011).

[58] C. Pfeiffer and A. Grbic, Millimeter-wave transmitarrays for wavefront and polarization control, IEEE Trans. Microwave Theory Tech. 61, 4407 (2013).

[59] F. Monticone, N. M. Estakhri, and A. Alù, Full Control of Nanoscale Optical Transmission with a Composite Metascreen, Phys. Rev. Lett. 110, 203903 (2013).

[60] A. N. Gentselev, B. G. Goldenberg, and S. A. Kuznetsov, Russian Federation Patent No. RU2548945 (2015).

[61] S. A. Kuznetsov, A. N. Gentselev, and S. G. Baev, Implementation of high-pass subterahertz filters using highaspect-ratio polimeric structures, Optoelectron. Instrum. Data Process. 53, 88 (2017).

[62] S. A. Kuznetsov, A. V. Arzhannikov, V. V. Kubarev, P. V. Kalinin, M. Sorolla, M. Navarro-Cia, M. Aznabet, M. Beruete, F. Falcone, Yu. G. Goncharov, B. P. Gorshunov, A. V. Gelfand, and N. I. Fedorinina, Development and characterization of quasi-optical mesh filters and metastructures for subterahertz and terahertz applications, Key Eng. Mat. 437, 276 (2010).

[63] V. Saile, U. Wallrabe, O. Tabata, and J. G. Korvink, in Advanced Micro \& Nanosystems (Wiley-VCH, New York, 2009). 